\title{
Cosmic Censorship: As Strong As Ever
}

\author{
Patrick R. Brady, ${ }^{1}$ Ian G. Moss, ${ }^{2}$ and Robert C. Myers ${ }^{3}$ \\ ${ }^{1}$ Theoretical Astrophysics 130-33, California Institute of Technology, Pasadena, California 91125 \\ ${ }^{2}$ Department of Physics, University of Newcastle Upon Tyne, NE1 7RU United Kingdom \\ ${ }^{3}$ Institute of Theoretical Physics, University of California, Santa Barbara, California 93117
}

(Received 12 January 1998)

\begin{abstract}
Spacetimes which have been considered counterexamples to strong cosmic censorship are revisited. We demonstrate the classical instability of the Cauchy horizon inside charged black holes embedded in de Sitter spacetime for all values of the physical parameters. The relevant modes that maintain the instability, in the regime which was previously considered stable, originate as outgoing modes near the black-hole event horizon. This same mechanism is also relevant for the instability of Cauchy horizons in other proposed counterexamples to strong cosmic censorship. [S0031-9007(98)05837-2]
\end{abstract}

PACS numbers: 04.20.Dw

As demonstrated by the elegant theorems of Hawking and Penrose [1], spacetime singularities are unbiquitous features of general relativity. Thus Einstein's theory itself impels us to search for a more fundamental theory of gravity in order to understand the physics of these extreme situations. The utility of general relativity in describing gravitational phenomena is maintained by cosmic censorship [2]. The latter is based on the common wisdom that singularities are not pervasive, and has been expressed in two forms: (1) Weak cosmic censorship states that, beginning with generic initial conditions, singularities only form in gravitational collapse hidden behind an event horizon. (2) Strong cosmic censorship states that the evolution of generic initial data will always produce a globally hyperbolic spacetime.

Thus the weak form of the conjecture suggests singularities are always hidden inside of black holes, invisible to distant observers. The strong form indicates that singularities only appear on spacelike or null surfaces, and so are hidden from all observers; i.e., the only way to examine a spacetime singularity is to run into it. At present, no rigorous theorems have been established to prove either of these conjectures; rather the evidence for (or against) cosmic censorship comes from our experience in solving Einstein's equations. Of the two conjectures, weak cosmic censorship enjoys a better "bill of health" [3]. Strong cosmic censorship seems to have run afoul of certain counterexamples in which timelike singularities develop for a (small but) finite range of physical parameters [4]. Strong cosmic censorship and these examples are the focus of this Letter. We will demonstrate that a more complete analysis of the latter solutions shows that they do not provide counterexamples to strong cosmic censorship. It is worth emphasizing that the failure of the strong form of cosmic censorship would indicate that the predictability of the Einstein equations can be lost in regions of spacetime where observers encounter no extreme gravitational fields.

Solutions of Einstein's equations which have timelike singularities hidden inside event horizons are familiar; both Reissner-Nordström and Kerr-Newman black holes belong to this class. In general, there is a Cauchy horizon $(\mathrm{CH})$ associated with a timelike singularity. The $\mathrm{CH}$ is a null hypersurface which marks the limit of the evolution of the solution from some initial time slice; that is, observers that cross the $\mathrm{CH}$ enter a region in which past directed null geodesics may terminate on the singularity. The ReissnerNordström solution, given by setting $\Lambda=0$ in Eqs. (1) and (2), is the archetypical example of this situation. The solution has two horizons at $r_{ \pm}=M \pm \sqrt{M^{2}-Q^{2}}$ determined by solving $f(r)=0$. The smaller horizon $r_{-}$is the $\mathrm{CH}$. The solution can be analytically extended to include $r=0$, which is then the locus of a timelike singularity. However, many extensions to $r<r_{-}$are possible corresponding to alternative boundary conditions at the origin. Thus, this elementary solution of Einstein equations is not globally hyperbolic.

Nevertheless, the Reissner-Nordström metric should not be considered a counterexample to strong cosmic censorship. Building on the initial observation by Penrose [5] that the $\mathrm{CH}$ is a surface of infinite gravitational blueshift, it has been demonstrated that the $\mathrm{CH}$ is unstable to linear gravitational and electromagnetic perturbations [6,7]. Further investigations have demonstrated that the $\mathrm{CH}$ is transformed into a null, scalar curvature singularity when full nonlinear evolution is considered [8-10]. The essential feature responsible for the instability is the same in all of these analyses: small time-dependent perturbations originating outside the black hole are gravitationally blueshifted as they propagate inwards parallel to the $\mathrm{CH}$. The locally measured flux of these perturbations grows without bound as the $\mathrm{CH}$ is approached along timelike geodesics.

This situation changes if the charged black hole is immersed in de Sitter space by the introduction of a positive cosmological constant, $\Lambda$. The metric takes the form

$$
\begin{gathered}
d s^{2}=-f(r) d t^{2}+\frac{d r^{2}}{f(r)}+r^{2}\left(d \theta^{2}+\sin ^{2} \theta d \phi^{2}\right), \\
f(r)=1-\frac{2 M}{r}+\frac{Q^{2}}{r^{2}}-\frac{\Lambda r^{2}}{3} .
\end{gathered}
$$


In this solution, there are three horizons corresponding to the positive solutions of $f(r)=0$; we label them $r_{3} \leq$ $r_{2} \leq r_{1}$ where $r_{3}$ denotes the Cauchy horizon, $r_{2}$ is the event horizon, and $r_{1}$ is the radius of the cosmological horizon. Thus, one again finds an inner $\mathrm{CH}$ and a timelike singularity at $r=0$. In terms of global structure, the main modification is at large radius where the spacetime is asymptotically de Sitter rather than flat. As a result, the standard blueshift argument of Penrose is slightly modified. Radially infalling radiation which propagates along the $\mathrm{CH}$ originates in the asymptotic region close to the cosmological horizon. Consequently, such radiation is redshifted as it falls away from the cosmological horizon as well as being blueshifted at the $\mathrm{CH}$; there is a competition of these two effects in determining the corresponding flux of radiation at the $\mathrm{CH}$. For a limited range of physical parameters (corresponding to near-extremal black holes), one finds that the cosmological redshift dominates and a finite flux is produced $[11,12]$. Thus this mechanism is ineffective in destabilizing the $\mathrm{CH}$.

The essential point of the present Letter is that, in this latter situation, one must extend the analysis to also consider outgoing modes which originate from close to the event horizon. These modes are scattered by the curvature to produce an additional influx along the $\mathrm{CH}$. There is again a competition of a redshift in climbing away from the event horizon and a blueshift in falling towards the $\mathrm{CH}$, but, in this case, the latter always dominates to produce a diverging flux at the $\mathrm{CH}$. Generically, this effect is subdominant in comparison to the flux due to the infalling modes; however, it persists into the regime where the latter only yield a finite flux. This argument, which is made precise below, demonstrates that the $\mathrm{CH}$ remains unstable over the entire range of physical parameters, and that Reissner-Nordström-de Sitter black holes are not counterexamples to strong cosmic censorship.

To begin a quantitative discussion, we transform the metric (1) to null coordinates

$$
d s^{2}=-f(r) d v d u+r^{2}\left(d \theta^{2}+\sin ^{2} \theta d \phi^{2}\right),
$$

where $u=t-r_{*}$ and $v=t+r_{*}$ are defined in terms of the tortoise radial coordinate

$$
r_{*}=\int d r / f(r) .
$$

These coordinates are illustrated in Fig. 1. The mainpoints to note are $v=\infty$ on the ingoing sheets of the cosmological and the inner horizons, and $u=\infty$ on the outgoing sheet of the black-hole event horizon. The various blueshift and redshift effects discussed above are controlled by the surface gravities of the respective horizons. The latter are defined by

$$
\kappa_{i}=\frac{1}{2}\left|\frac{d f}{d r}\right|_{r=r_{i}},
$$

where $1 \leq i \leq 3$.

In this spacetime, we consider the evolution of linearized perturbations denoted as $\Phi$. The field $\Phi$ satisfies a wave

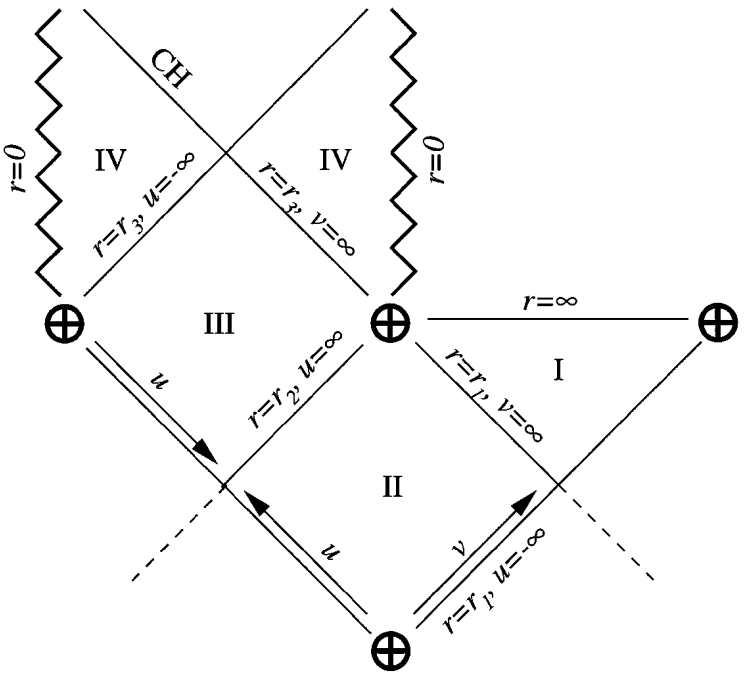

FIG. 1. A portion of the Penrose conformal diagram for the Reissner-Nordström-de Sitter black-hole spacetime. Regions II and III correspond to the exterior and interior of the black hole, respectively, separated by the event horizon at $r=r_{2}$. See Ref. [4] for a detailed description of the spacetime geometry.

equation, which can be reduced to a one-dimensional scattering problem, e.g., see Eq. (10) below, by virtue of the spherical symmetry and static nature of the background spacetime. If the evolution produces a diverging flux of radiation as measured by observers at the $\mathrm{CH}$, the result is interpreted as indicating the $\mathrm{CH}$ is unstable. The flux received by any observer is proportional to the square of the amplitude

$$
\mathcal{F}=\Phi_{, \alpha} u^{\alpha},
$$

where $u^{\alpha}$ is the observer's four-velocity.

Now the essential features of the linear perturbation analysis can be summarized by the following argument. First, reasonable initial conditions must be determined for perturbations in the vicinity of the cosmological and event horizons. Generally, observers crossing the cosmological horizon will measure a finite flux. Considering Eq. (6) for a radially moving observer, one shows that $\Phi$ must satisfy

$$
\Phi_{, v} \sim e^{-\kappa_{1} v}
$$

as $v \rightarrow \infty$. This determines the behavior of the initial ingoing modes. Observers falling into the black hole should see a finite flux of radiation at the event horizon. Similarly, this requires that the variation of the field satisfy

$$
\Phi_{, u} \sim e^{-\kappa_{2} u}
$$

as $u \rightarrow \infty$ in both regions II and III, fixing the initial conditions for the outgoing modes. The evolution of these outgoing modes will result in backscattering, adding an extra contribution to the influx along the $\mathrm{CH}$. This additional flux may be estimated by observing that the backscattering occurs roughly on a $v-u=$ const surface; i.e., the 
effective potential falls off very rapidly near the event and Cauchy horizons, and so in this process the $u$ dependence of Eq. (8) is converted to a $v$ dependence. Therefore, the total amplitude measured by observers crossing the $\mathrm{CH}$ takes the form

$$
\mathcal{F} \sim e^{\kappa_{3} v}\left(e^{-\kappa_{1} v}+\text { const } \times e^{-\kappa_{2} v}\right) .
$$

The first term above, due to the ingoing modes, produces a divergent result for $\kappa_{3}>\kappa_{1}$, which is satisfied except for near-extremal black holes $[11,12]$. The backscattered contribution diverges for $\kappa_{3}>\kappa_{2}$, which is valid whenever $r_{3} \neq r_{2}$. Therefore the second flux ensures that the $\mathrm{CH}$ is generally unstable. It should be noted that over most of the range of physical parameters $\kappa_{1}>\kappa_{2}$, so the backscattered term is subdominant and neglecting the outgoing modes still yields quantitatively correct results. It is only in the regime previously thought to be stable, i.e., $r_{3} \simeq r_{2}$, that the importance of the outgoing modes manifests itself.

While the previous argument may appear simplistic, the final result for the amplitude (9) is supported by our detailed analysis of the linear instability of the Cauchy horizon. Our approach was threefold: extending the null fluid model of [11] and the mode analysis of [12] to incorporate backscattering, and making numerical investigations to confirm the latter analytic results. The details of this work will be presented elsewhere, but here we discuss the new result revealed by the mode analysis. This mechanism for the instability of the $\mathrm{CH}$ arises purely from modes confined to the interior of the black hole, i.e., region III of Fig. 1

The equations governing the metric and electromagnetic perturbations of a Reissner-Nordström-de Sitter black hole have been worked out in detail in [13], where it was shown that they reduce to four scalar wave equations. The perturbation fields $\Phi$ are decomposed into eigenmodes of frequency $\omega$ and spherical harmonics, which satisfy

$$
\left(\frac{d^{2}}{d r_{*}^{2}}+\omega^{2}\right) \hat{\Phi}\left(\omega, r_{*}\right)=V\left(r_{*}\right) \hat{\Phi}\left(\omega, r_{*}\right)
$$

(where angular eigenvalues will be suppressed throughout). The details of the potential depend on the type of perturbation [13], e.g., for axial perturbations

$$
V=f(r)\left[\frac{a}{r^{2}}+\frac{b}{r^{3}}+\frac{c}{r^{4}}\right]
$$

where Eq. (2) gives $f(r)$, Eq. (4) determines $r\left(r_{*}\right)$, and $a, b, c$ are certain constants. An important general feature is that the potential is always analytic in both $\exp \left(-\kappa_{3} r_{*}\right)$ and $\exp \left(\kappa_{2} r_{*}\right)$ throughout region III. It is useful to introduce a basis of mode solutions of Eq. (10): $\overleftarrow{\Phi}\left(\omega, r_{*}\right)$ and $\vec{\Phi}\left(\omega, r_{*}\right)$ normalized to satisfy

$$
\begin{aligned}
& \overleftarrow{\Phi}\left(\omega, r_{*}\right) \rightarrow e^{-i \omega r_{*}} \\
& \vec{\Phi}\left(\omega, r_{*}\right) \rightarrow e^{i \omega r_{*}}
\end{aligned} \text { as } r_{*} \rightarrow-\infty .
$$

These modes represent initially ingoing and outgoing waves, respectively, in the black-hole interior. The full time-dependent solution can now be written as

$$
\begin{aligned}
\Phi\left(t, r_{*}\right)=\int_{-\infty}^{\infty} \frac{d \omega}{2 \pi}\left[\overleftarrow{W}(\omega) \overleftarrow{\Phi}\left(\omega, r_{*}\right)\right. \\
\left.+\vec{W}(\omega) \vec{\Phi}\left(\omega, r_{*}\right)\right] e^{-i \omega t}
\end{aligned}
$$

with the functions $W(\omega)$ being determined by the initial data.

Perturbations falling in across the event horizon from the exterior would fix $\overleftarrow{W}(\omega)$. These would be analyzed as in Ref. [13], and we do not consider them further here. Instead, we focus on outgoing perturbations which would arise from the surface to the star which collapses to form the black hole. These would be the perturbations determining $\vec{W}(\omega)$. The asymptotic behavior of the field given in Eq. (8) implies that $\vec{W}(\omega)$ has a pole at $\omega=-i \kappa_{2}$.

As above, we wish to determine the flux of radiation measured by an observer crossing the $\mathrm{CH}$, and so must calculate the amplitude $\mathcal{F}$ defined in Eq. (6). The part of the amplitude which may be divergent at the $\mathrm{CH}$ is

$$
\mathcal{F} \sim e^{\kappa_{3} v} \Phi_{, v} .
$$

Now the initially outgoing modes of Eq. (12) are dispersed by the potential between the two horizons so that as $r_{*} \rightarrow \infty$

$$
\vec{\Phi} \rightarrow A(\omega) e^{i \omega r_{*}}+B(\omega) e^{-i \omega r_{*}} .
$$

It is the behavior of the reflected waves that are relevant to our discussion, thus

$$
\overrightarrow{\mathcal{F}} \sim e^{\kappa_{3} v} \int_{-\infty}^{\infty} d \omega \omega \vec{W}(\omega) B(\omega) e^{-i \omega v} .
$$

The integral is computed by closing the contour in the lower half-plane and using the residue theorem. The dominant contribution to the flux comes from the pole nearest to the real axis. Using arguments similar to those in Ref. [7], one shows that $\omega B(\omega)$ is analytic in the strip $\left[-i \kappa_{3}, i \kappa_{2}\right]$. Hence, the pole in $\vec{W}(\omega)$ at $-i \kappa_{2}$ provides the leading contribution, that is,

$$
\overrightarrow{\mathcal{F}} \sim e^{\left(\kappa_{3}-\kappa_{2}\right) v}\left\{-i \kappa_{2} B\left(-i \kappa_{2}\right) \operatorname{Res}\left[\vec{W}\left(-i \kappa_{2}\right)\right]\right\} .
$$

As discussed above, it is easy to show that $\kappa_{3}>\kappa_{2}$ provided that $r_{3} \neq r_{2}$; therefore, $\overrightarrow{\mathcal{F}}$ always diverges as $v \rightarrow \infty$ provided $B\left(-i \kappa_{2}\right)$ is nonzero. While it seems unlikely that $B\left(-i \kappa_{2}\right)$ would vanish, we have verified it numerically.

The evolution of scalar waves on the spherical blackhole de Sitter spacetimes has been considered in [14]. It is straightforward to apply the same numerical techniques to the fields $\Phi$ above, except inside the black-hole horizon. Reinstating the time dependence in the scattering equations (10), the wave equations may be written as

$$
\Phi_{, u v}=-\frac{1}{4} V(r) \Phi \text {. }
$$

We use a characteristic evolution scheme to solve these equations, so the initial data is supplied on an ingoing null hypersurface $v=0$, and the event horizon of the 
black hole (in reality a very large positive value of $u$ ). The initial data corresponds to what can reasonably be expected from a collapsing star. Near the event horizon the field is presumed to be analytic in a Kruskalized coordinate tailored to that horizon. Thus

$$
\Phi(u, v=0) \simeq \Phi_{0}+\Phi_{1} e^{-\kappa_{2} u}+\ldots,
$$

which reproduces the dependence of Eq. (8). On the event horizon the field was taken to decay exponentially with advanced time; the precise form was motivated by considerations of tails of gravitational collapse in the external field of the black hole [14]. However, the results are insensitive to the details of these boundary conditions.

The results of the numerical integration are consistent with the scattering analysis described above. We find the rate of decay of the field satisfies

$$
\Phi_{, v} \propto e^{-\sigma v}
$$

along surfaces of constant $u$ crossing the $\mathrm{CH}$, where the decay constant $\sigma$ was found to equal $\kappa_{2}$ within numerical errors. For example, in a calculation with $M=1.0, Q=1.000015$, and $\Lambda=10^{-4}, \sigma$ was equal to $\kappa_{2}$ to an accuracy of $\sim 0.035 \%$. Moreover, the decay of the perturbations at the horizon was also found to be independent of the angular eigenvalues, in contrast to the results obtained for wave evolution in the exterior region [14]. Together these calculations show that the instability of the $\mathrm{CH}$ will, in fact, generally result from modes entirely confined to the interior of the black hole.

In this Letter, we have shown that the $\mathrm{CH}$ of the Reissner-Nordström-de Sitter black hole is unstable to linear perturbations over the entire range of physical parameters. This should imply that $\mathrm{CH}$ instability also arises in the full nonlinear evolution. The significant new contribution was identified as arising from the backscattering of outgoing perturbations emerging near the event horizon. The physical origin of such an outflux is nothing more than the collapsing star which forms the black hole, and must surely be present. The backscattered flux extends the instability of the $\mathrm{CH}$ through the regime previously thought to be stable. Furthermore, our analysis readily extends to the other proposed counterexamples to strong cosmic censorship, such as accelerating black holes [15], or rotating black holes in de Sitter space [16]. Once again backscattering of initially outgoing modes provokes the instability of the $\mathrm{CH}$ for otherwise stable configurations. Thus, one may conclude that there are no known counterexamples to strong cosmic censorship within classical general relativity coupled to reasonable matter.

P.R. B. is supported by the Sherman Fairchild Foundation Inc. and NSF Grant No. AST-9417371. R. C. M. is supported by NSERC of Canada, and by NSF Grant No. PHY94-07194. We also acknowledge useful conversations with D. M. Eardley and K. Thorne.

[1] S. W. Hawking and R. Penrose, Proc. R. Soc. London A 314, 529 (1970).

[2] R. Penrose, Riv. Nuovo Cimento I 1, 252 (1969); in General Relativity, an Einstein Centenary Survey, edited by S. W. Hawking and W. Israel (Cambridge University Press, Cambridge, 1979).

[3] See the recent review in R.M. Wald, e-print gr-qc/ 9710068. Note, however, that less optimistic prognoses have also been given by others, e.g., S. W. Hawking and K. Thorne, as reported in New York Times, 12 February 1997, and D. M. Eardley (private communication).

[4] See the recent review in C. Chambers, e-print gr-qc/ 9709025.

[5] R. Penrose, in Battelle Rencontres, edited by C. de Witt and J. Wheeler (W. A. Benjamin, New York, 1968), p. 222.

[6] R. A. Matzner, N. Zamorano, and V.D. Sandberg, Phys. Rev. D 19, 2821 (1979).

[7] S. Chandrasekhar and J. Hartle, Proc. R. Soc. London A 384, 301 (1982).

[8] E. Poisson and W. Israel, Phys. Rev. D 41, 1796 (1990).

[9] A. Ori, Phys. Rev. Lett. 67, 789 (1991).

[10] P. R. Brady and J.D. Smith, Phys. Rev. Lett. 75, 1256 (1995).

[11] P. R. Brady and E. Poisson, Classical Quantum Gravity 9, 121 (1992).

[12] F. Mellor and I. G. Moss, Classical Quantum Gravity 9, L43 (1992).

[13] F. Mellor and I. G. Moss, Phys. Rev. D 41, 403 (1990).

[14] P. R. Brady, C. M. Chambers, W. Krivan, and P. Laguna, Phys. Rev. D 55, 7538 (1997).

[15] G. T. Horowitz and H. J. Sheinblatt, Phys. Rev. D 55, 650 (1997).

[16] C. M. Chambers and I.G. Moss, Classical Quantum Gravity 11, 1034 (1994); C. M. Chambers, Ph.D. thesis, University of Newcastle Upon Tyne, 1995. 